\title{
Erratum
}

\section{The metallicity dependence of the Cepheid PL-relation}

\author{
M. A. T. Groenewegen ${ }^{1}$, M. Romaniello ${ }^{2}$, F. Primas ${ }^{2}$, and M. Mottini ${ }^{2}$ \\ 1 Instituut voor Sterrenkunde, PACS-ICC, Celestijnenlaan 200B, 3001 Leuven, Belgium \\ e-mail: groen@ster . kuleuven.ac.be \\ 2 ESO, Karl Schwarzschild straße 2, 85748 Garching, Germany
}

A\&A, 420, 655-663 (2004), DOI: 10.1051/0004-6361:20035926

Key words. stars: distances - Cepheids - errata, addenda

Equation (1) in the paper "The metallicity dependence of the Cepheid PL-relation" by Groenewegen et al. (2004, A\&A, 420, $655)$ contains an unfortunate typographical error: all terms " $\log [\mathrm{Fe} / \mathrm{H}]$ " should read " $[\mathrm{Fe} / \mathrm{H}]$ ".

The correct formula is thus:

$M=\alpha_{1}+\alpha_{2}[\mathrm{Fe} / \mathrm{H}]+\alpha_{3}[\mathrm{Fe} / \mathrm{H}]^{2}$

$$
\begin{aligned}
& +\alpha_{4}\left(1+\frac{\alpha_{5}}{\alpha_{4}}[\mathrm{Fe} / \mathrm{H}]+\frac{\alpha_{6}}{\alpha_{4}}[\mathrm{Fe} / \mathrm{H}]^{2}\right) \log P_{0} \\
& +\alpha_{7}\left(1+\frac{\alpha_{8}}{\alpha_{7}}[\mathrm{Fe} / \mathrm{H}]+\frac{\alpha_{9}}{\alpha_{7}}[\mathrm{Fe} / \mathrm{H}]^{2}\right)\left(\log P_{0}\right)^{2} \\
& +\beta\left(m_{1}-m_{2}\right)+\Delta_{\mathrm{LMC}} x_{\mathrm{LMC}}+\Delta_{\mathrm{SMC}} x_{\mathrm{SMC}},
\end{aligned}
$$

All results remain unchanched as the fitting was performed using the correct formula. 